\title{
Electronic and Nuclear Factors in Intramolecular Charge and Excitation Transfer
}

Department of Energy Grant DE-FG05-92ER14310

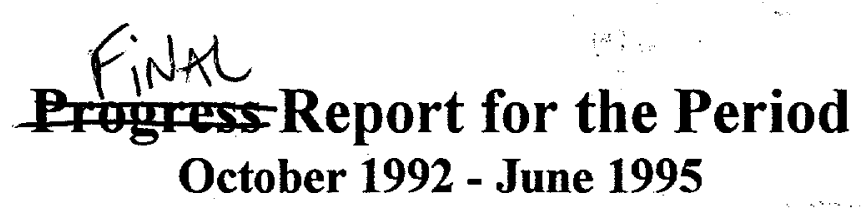

Piotr Piotrowiak

University of New Orleans 


\section{DISCLAIMER}

This report was prepared as an account of work sponsored by an agency of the United States Government. Neither the United States Government nor any agency thereof, nor any of their employees, make any warranty, express or implied, or assumes any legal liability or responsibility for the accuracy, completeness, or usefulness of any information, apparatus, product, or process disclosed, or represents that its use would not infringe privately owned rights. Reference herein to any specific commercial product, process, or service by trade name, trademark, manufacturer, or otherwise does not necessarily constitute or imply its endorsement, recommendation, or favoring by the United States Government or any agency thereof. The views and opinions of authors expressed herein do not necessarily state or reflect those of the United States Government or any agency thereof. 


\section{DISCLAIMER}

Portions of this document may be illegible in electronic image products. Images are produced from the best available original document. 


\section{Table of contents:}

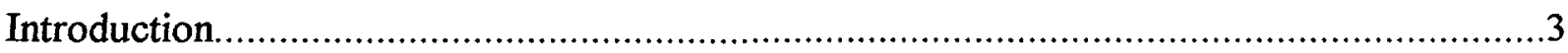

Dynamics and energetics of ion pairing with a charge separated species..............................4

The role of MO and vibrational mode symmetry in intramolecular excitation transfer.............14

Zwitter-ionic and biradicaloid excited states of twisted olefins............................................19

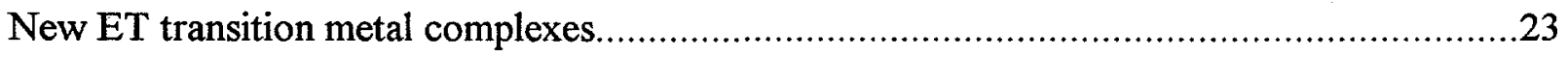

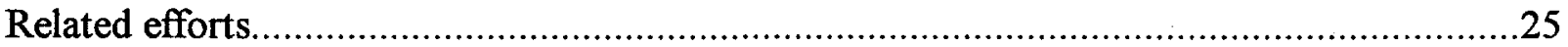

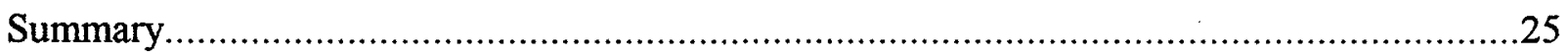

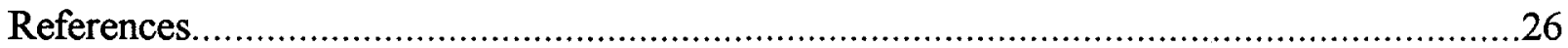

List of publications and presentations of the DOE funded research....................................27 


\section{Introduction}

In this report we describe the October 1992 through June 1995 progress our Department of Energy supported projects gathered under the broad title "Electronic and Nuclear Factors in Intramolecular Charge and Excitation Transfer". As the title indicates, our work is devoted to developing a better understanding of the fundamental factors controlling the rate and efficiency of photoinduced electron and electronic energy transfer processes. The two topics of special focus are: 1) the electrolyte effects in intramolecular electron transfer; 2) the role of the MO, as well as vibrational mode, symmetry in determining the donor-acceptor electronic coupling.

In the first chapter entitled "Dynamics and Energetics of Ion Pairing with a Charge Separated Species" the use of transient triplet charge transfer absorption bands as highly sensitive probes of ion pairing dynamics and energetics is described. Electrolyte induced spectral shifts approaching $1 \mathrm{eV}$ were recorded. We demonstrate that thanks to their long lifetimes triplet CT states allow to extend the study of electrolyte effects to $10^{-3}$ lower concentrations and much less polar media than it was possible with the standard fluorescent probes. The completed synthesis of donor-acceptor compounds capable of intramolecular ion association is also reported.

The second chapter entitled "The Role of Molecular Orbital and Vibrational Mode Symmetry in Intramolecular Excitation Transfer" reports the high resolution molecular beam work on the electronic coupling and excitation transfer in rigid spirobifluorenes with symmetry forbidden donor-acceptor interactions. The dependence of the energy transfer on excitation of symmetry breaking vibrational modes, as well as the unusually clear transition from the "small molecule" to "large molecule" behavior, are described.

The chapter entitled "Zwitter-Ionic and Biradicaloid Excited States of Twisted Olefins" gives a preliminary report of the observation of an unusually long lived zwitter-ionic excited singlet state and a biradicaloid triplet state of a twisted olefin, biphenanthrenylidene.

In the last chapter, "New ET Transition Metal Complexes", we describe the recent synthesis of a new family of bridging and terminal ligands which will serve as building blocks for a variety of binuclear complexes and oligomeric materials. We also report the interesting x-ray structure of a new mononuclear ruthenium complex prepared in our laboratory.

Results which have been described in the attached reprints and manuscripts are only briefly summarized in the Report. The most recent unpublished results are described in more detail. 


\section{Dynamics and energetics of ion pairing with a charge separated species}

Diffusive relaxation of ions and ion association can play an important role in controlling the rate and efficiency of electron transfer processes ${ }^{1}$. Recent data of Tiede et al. indicate that the relaxation of ions embedded in the protein matrix contributes to the stabilization of the charge separated state in photosynthetic reaction centers. ${ }^{2}$ Electrolyte effects are important in most inorganic and organometallic ET systems which inevitably contain counter-ions.

The prior work on ionic relaxation following generation of a charge separated species in solutions of electrolytes (Huppert, Maroncelli) ${ }^{3}$ utilized commonly available probes of solvation dynamics, such as coumarin, and the dynamic red shifts of the CT singlet fluorescence were monitored. The disadvantage of the fluorescent probes lies in the short, 1-3 ns lifetime of the emissive singlet state. As a result, only concentrated solutions of electrolytes $(\sim 100 \mathrm{mM})$ can be investigated, if the diffusional relaxation of the ions is to compete with the short lifetime of the probe state. Such high salt concentrations can be obtained only in reasonably polar media, and hence acetonitrile is perhaps the best studied solvent. Unfortunately, the high dielectric constant of the medium attenuates the electrostatic interaction between the probe and the electrolyte, considerably lowering the magnitude of the spectral shift, and of the ionic stabilization of the charge separated state.

We proposed to use as probes molecules with long lived triplet charge separated states, and to monitor the dynamic blue shift of the transient absorption from the CT triplet to a higher local triplet state, rather than the red shift of the emission (see the diagram below).

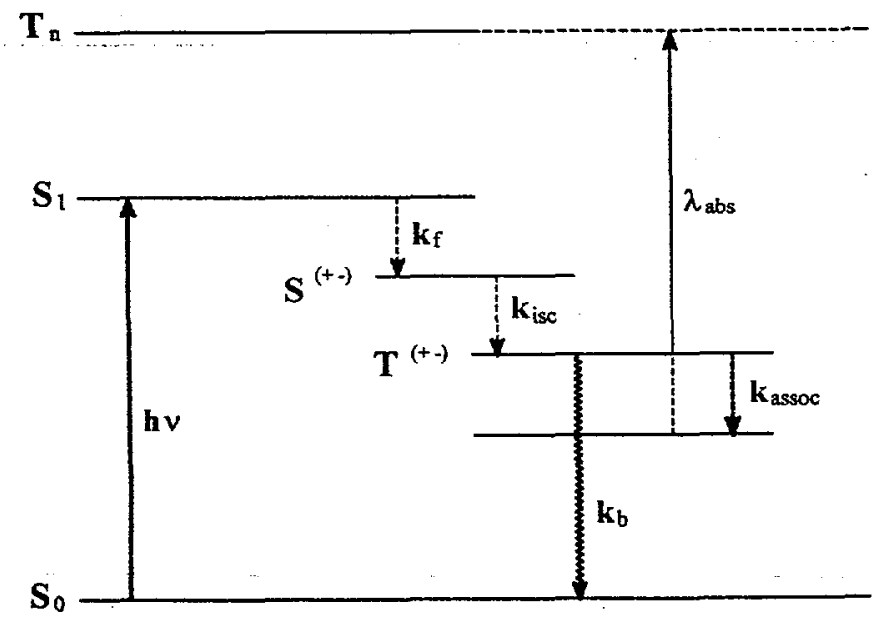

Figure 1. Simplified energy diagram of the states involved in the photoinduced charge separation of p-ANTP and subsequent ion association. 
The $p$-aminonitrobiphenyl (p-ANBP) and $p$-aminonitroterphenyl (p-ANTP) were selected as probes because some aspects of their photophysics are relatively well characterized (the pANBP is known to form charge separated triplet state within $\sim 3 \mathrm{~ns}$ of photoexcitation), ${ }^{4}$ and they are very easy to prepare ( 1 or 2 -step synthesis). The discussion concentrates on the p-ANTP, which was found to be somewhat more sensitive to the changes of environment than p-ANBP. All qualitative findings apply also to p-ANBP. Majority of the initial work was done in THF, $\varepsilon=$ 7.6 , because it provides a combination of moderate polarity and good solubility of a large number of salts. THF is also one of the most popular solvents for electron transfer studies in inorganic complexes.

The CT triplet state of p-ANTP exhibits strong absorption in the visible and near IR, with $\lambda_{\max }$ of $990 \mathrm{~nm}$ and $\varepsilon_{990} \approx 40000 \mathrm{in} \mathrm{THF}$. The triplet state has a long lifetime of $3.1 \mu$ s, i.e. 1000times longer than the lifetime of fluorescent probes. ${ }^{5}$ The spectra are strongly solvatochromic.

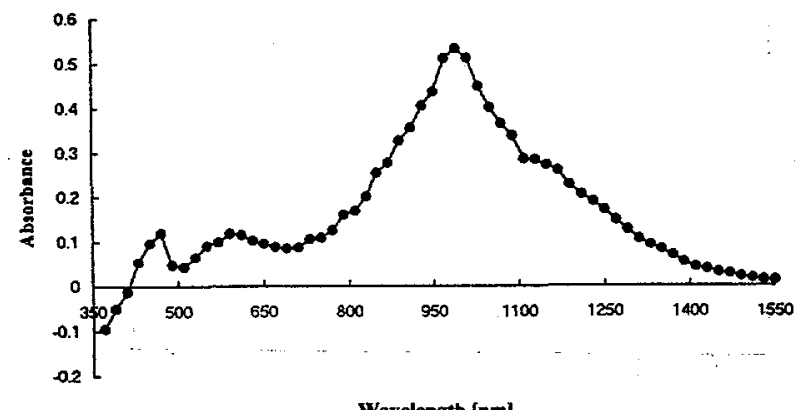

Wavelength [nw]

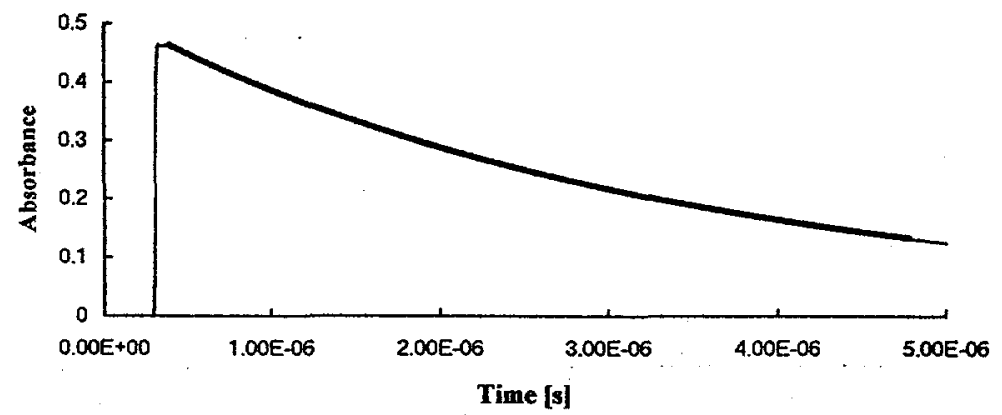

Figure 2. a) The triplet absorption spectrum of p-ANTP in neat THF, $355 \mathrm{~nm}$ excitation; b) transient absorption profile at $990 \mathrm{~nm}, \tau=3.1 \mu \mathrm{s}$. Note the excellent agreement between the data and the fit.

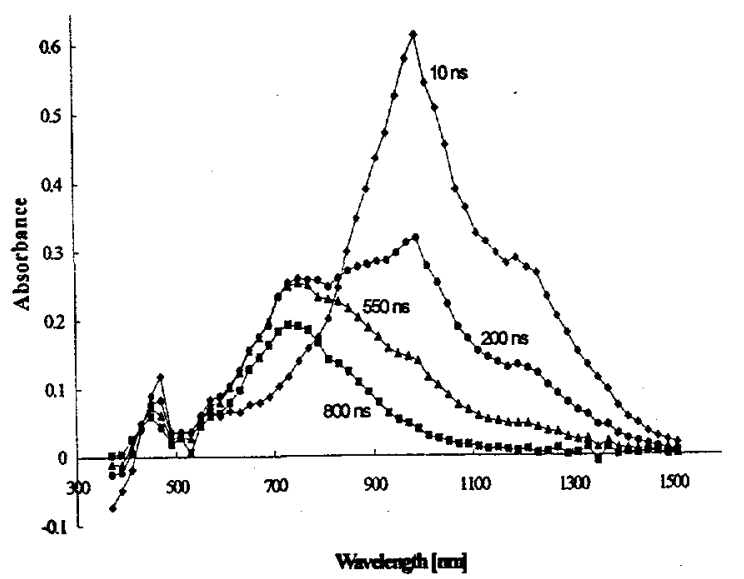

Figure 3. Time evolution of the triplet CT absorption of $0.1 \mathrm{mM} \mathrm{p}$-ANTP in THF in the presence of $1.0 \mathrm{mM}$ of TBABr, following $355 \mathrm{~nm}$ excitation. 
Upon addition of millimolar amounts of an electrolyte a dynamic blue shift of the triplet CT absorption of p-ANTP is observed (Fig. 3), as expected on the basis of the energy diagram in Fig. 1. Immediately after the laser pulse the spectrum is identical with the one obtained in neat THF. As the delay increases the spectrum evolves to a new equilibrium characterized by a much shorter wavelength $\left(\lambda_{\max }=710 \mathrm{~nm}\right.$ in the case of $\mathrm{TBABr}$ ). The equilibrium is completely shifted toward the associated form, with no residual peak at the neat THF $\lambda_{\max }$ value of $990 \mathrm{~nm}$. This is consistent with the magnitude of the shift $\left(-3600 \mathrm{~cm}^{-1}\right.$, or $10.3 \mathrm{kcal} / \mathrm{mol}$ for $\left.\mathrm{TBABr}\right)$, which implies that even at millimolar salt concentrations the association with the probe molecule is complete. It is important to note that there is no pre-association with the electrolyte in the ground state of p-ANTP (Fig. 3). As expected, there is no measurable effect of millimolar salt concentration on the fluorescence spectrum of p-ANTP, including its long wavelength CT band. This proves that no significant ion association with the singlet charge separated state can occur within its lifetime.

The above results demonstrate that transient $\mathrm{CT}$ absorption bands can be used as sensitive probes of ion pairing energetics and dynamics. The long lifetime of the probe state allows one to study highly dilute solutions of electrolytes in weakly polar (and nonpolar, see below) media.

Ion size dependence study: The study of the ion radius dependence of the magnitude of the electrolyte induced spectral shifts of the triplet CT state of p-ANTP focused on anions for two reasons: 1) they do not exhibit specific solvation or coordination in ether-like solvents; 2) it is possible to select a large set of anions with approximately spherical shapes. Cations display a

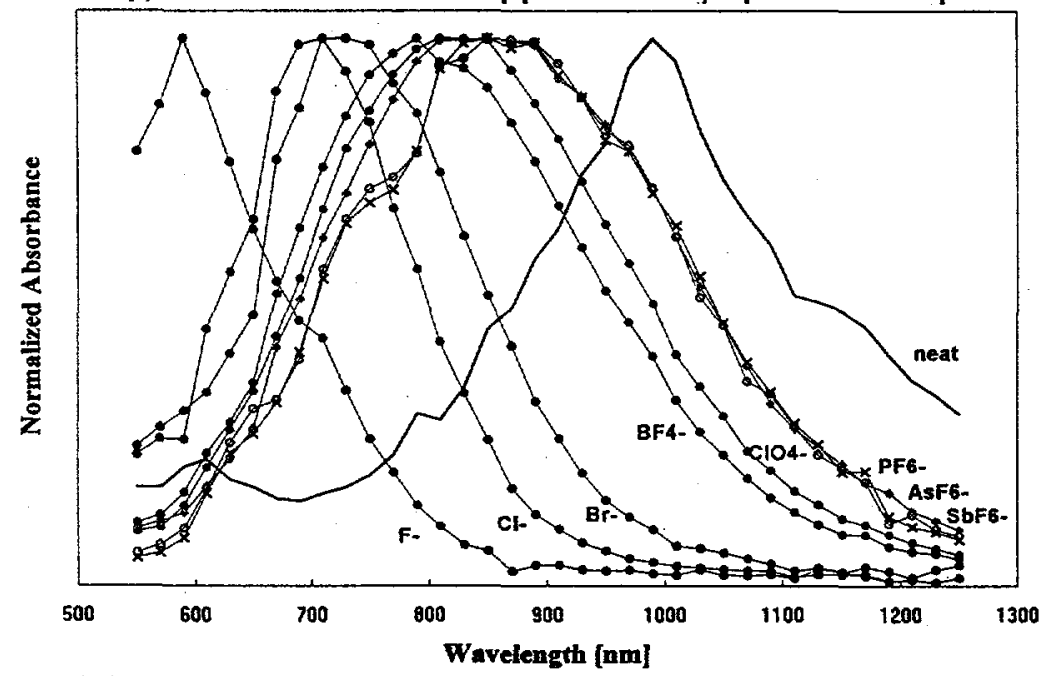

Figure 4. Salt induced shifts of the p-ANTP triplet CT absorption spectrum in THF. 
more complex behavior. Metal cations form tight coordination spheres in ether-like solvents. ${ }^{6}$ The floppy tetraalkylammonium cations have poorly defined shapes.

The selected anions ranged from $\mathrm{F}^{-}$to $\mathrm{SbF}_{6}^{-}$. The cation was kept constant and in all cases it was the tetrabutylammonium. The recorded fully equilibrated spectral maxima correlate well with the size of the ions, with smaller anions leading to larger blue shifts. The largest blue shift of $6850 \mathrm{~cm}^{-1}$ was obtained with the $\mathrm{F}^{-}$salt. It is more than 3-times larger that the largest electrolyte induced shifts observed with fluorescent probes. ${ }^{3}$ The smallest shift of $1670 \mathrm{~cm}^{-1}$ was recorded for the $\mathrm{AsF}_{6}{ }^{-}$and $\mathrm{SbF}_{6}{ }^{-}$anions. All measured shifts are reported in Table 1 and plotted versus $1 / r_{\text {anion }}$ below. The $I$ ion is missing from the list because it forms a CT complex with the charge separated species of p-ANTP. It is apparent from the plot that as the radius of the anion approaches infinity, the corresponding shift does not extrapolate to zero, but rather to a finite value of $\sim 1500 \mathrm{~cm}^{-1}$. This "baseline" value can be attributed to the contribution of the $\mathrm{TBA}^{+}$ cation, which should remain constant for all salts.

Table 1. Triplet p-ANTP spectral shifts induced by tetrabutylammonium salts.

\begin{tabular}{|c|c|c|c|}
\hline Anion & Radias $[\AA]$ & Shift $\left[\mathrm{cm}^{-1}\right]$ & Bandwidth $\left[\mathrm{cm}^{-1}\right]$ \\
\hline $\mathrm{F}^{-}$ & 1.19 & 6850 & $4701^{*}$ \\
$\mathrm{Cl}^{-}$ & 1.67 & 3980 & 3760 \\
$\mathrm{Br}^{-}$ & 1.82 & 3600 & 3760 \\
$\mathrm{BF}_{4}^{-}$ & 2.18 & 2560 & 4620 \\
$\mathrm{ClO}_{4}^{-}$ & 2.26 & 2240 & 4600 \\
$\mathrm{PF}_{6}^{-}$ & 2.96 & 1670 & 4670 \\
$\mathrm{AsF}_{6}^{-}$ & 2.87 & 1670 & 4760 \\
$\mathrm{SbF}_{6}^{-}$ & 3.26 & 1670 & 4760 \\
\hline
\end{tabular}

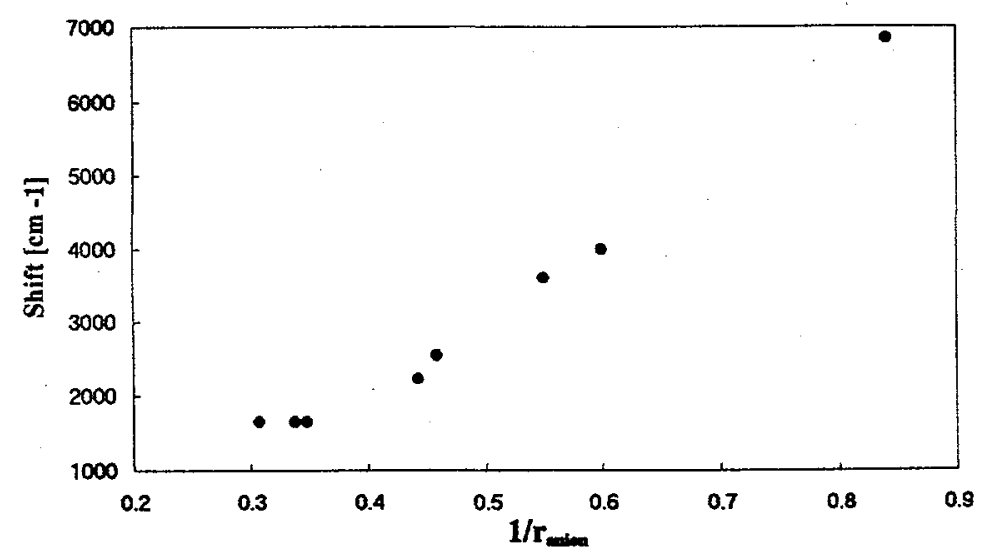

Figure 5. Spectral shifts from Table 1 plotted versus $1 / r_{\text {andon. }}$. 
The last point brings about the important question of the geometry (or geometries) of the associated complex containing the excited state molecule of p-ANTP and one or more ions of the salt. It is impossible to conclude on the basis of the presented data whether the association process takes place primarily at the $\mathrm{H}_{2} \mathrm{~N}^{\dagger}$ - side of the probe and is dominated by the anion of the salt, or at the $-\mathrm{NO}_{2}^{-}$side of the probe and is dominated by the cation of the salt. In order to unequivocally answer this question we propose time resolved Raman experiments on p-ANTP in the presence of electrolytes (see the Renewal Proposal).

To summarize, spectral shifts approaching $1 \mathrm{eV}$ have been observed in the presence of electrolytes $(0.92 \mathrm{eV}$ in the presence of $\mathrm{LiCL})$. The large magnitude of the shifts, and of the corresponding stabilization (or reorganization) energy, point out the importance of ion-pairing effects in controlling the energetics and dynamics of electron transfer in moderately polar media.

Concentration and temperature dependence studies: The dynamics of the spectral shifts of p-ANTP was studied over a broad range of salt concentrations. Only the $\mathrm{TBABr}$ data are presented here. It was found that the measured association rate varies linearly with the overall salt concentration, rather than as the $c^{1 / 2}$ dependence which might be expected on the basis of the Kohlrausch $\mathrm{law}^{7}$ for weakly dissociated electrolytes. Most salts are very weakly dissociated in THF, with dissociation constants of $1 \times 10^{-6}-1 \times 10^{-5}$. Therefore, even at concentrations as low as $1 \mathrm{mM}$ more than $90 \%$ of the salt remains undissociated. The small fraction of "free" ions has little influence on the overall dynamics of the association process which is dominated by the encounters between the charge separated species and ion pairs of the salt. Therefore, a linear

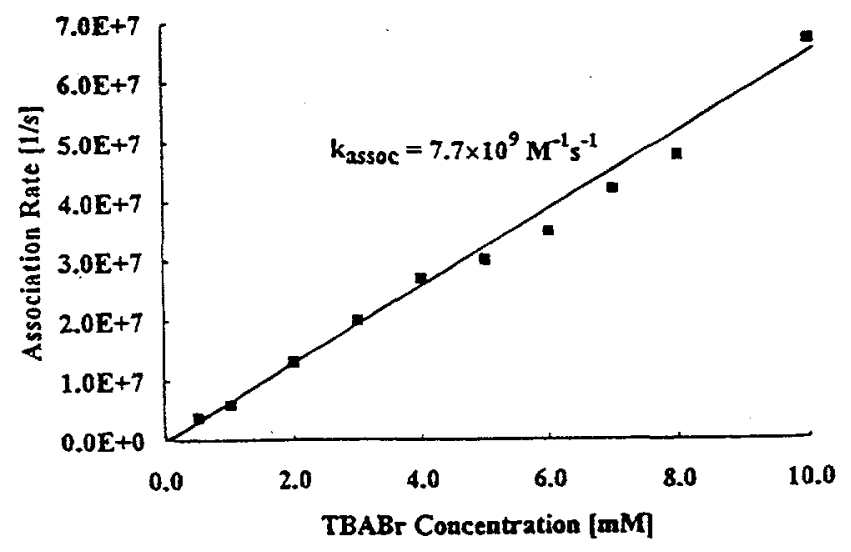

Figure 6. Dependence of the association rate on the concentration of TBABr in THF. 
dependence on the salt concentration should have been expected in the first place. The pseudounimolecular rate extracted from the TBABr data is $7.7 \times 10^{9} \mathrm{~s}^{-1}$ per 1 mole of the salt, i.e. very close to the diffusion controlled limit in THF. Departures from the simple linear behavior should occur both at very low $\left(<10^{-4} \mathrm{M}\right)$ and very high $\left(>10^{-1} \mathrm{M}\right)$ concentrations of a salt. This finding emphasizes that electrolyte effects in weakly polar media like THF have to be interpreted in terms of strong specific ion pairing, and that even at very low electrolyte concentrations $(<1 \mathrm{mM})$ the ionic atmosphere formalism utilized in some studies ${ }^{8}$ is inappropriate in these systems.

Systematic variable temperature studies of the pairing dynamics were undertaken in order to evaluate the activation energy of the process. $1 \mathrm{mM}$ THF solutions of two salts, the tetrabutylammonium bromide (TBABr) and tetrabutylammonium fluoride (TBAF), were studied over the temperature range of $-55^{\circ} \mathrm{C}$ to $25^{\circ} \mathrm{C}$. Interestingly, no significant changes in the $\lambda_{\max }$ and in the appearance of the spectra of the associated species were observed over this temperature range. The association process was monitored at $990 \mathrm{~nm}$ (the $\lambda_{\max }$ of the free probe), and at 700 and $590 \mathrm{~nm}$ (the equilibrium $\lambda_{\max }$ in the presence of TBABr and TBAF, respectively). The temperature was varied in small increments of 3 to $5^{\circ} \mathrm{C}$.
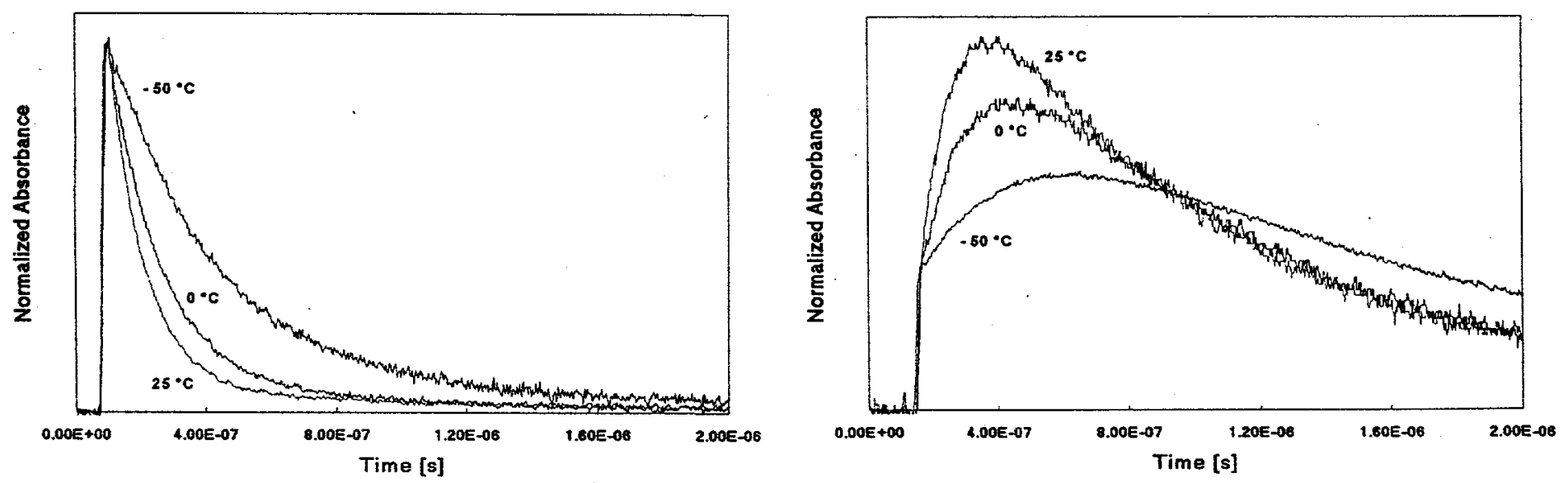

Figure 7. a) Temperature dependent p-ANTP transient absorption profiles at $990 \mathrm{~nm}$ (1 mM TBABr in THF);

b) temperature dependent p-ANTP transient absorption profiles at $700 \mathrm{~nm}(1 \mathrm{mM} \mathrm{TBABr}$ in THF).

The obtained plots exhibit remarkable linearity and attest to the reliability of the data. The $\mathrm{TBABr}$ and TBAF lines obtained from the $990 \mathrm{~nm}$ data (corresponding to the decay of the free probe) are nearly parallel and yield activation energies of 2.3 and $2.4 \mathrm{kcal} / \mathrm{mol}$ respectively. These values of $\mathrm{E}_{\mathrm{a}}$ are in the excellent agreement with a diffusion controlled process. Indeed, the slope 
of the lines can be reproduced to within $5 \%$ by using the empirical equation for the temperature dependence of the viscosity of THF. The rates for the TBAF are $20 \%$ faster than for $\mathrm{TBABr}$ over the entire temperature range. This agrees well with a diffusion controlled process which should be faster for the smaller $\left(\mathrm{TBA}^{+}, \mathrm{F}\right)$ ion pair.

The TBABr and TBAF lines obtained at 700 and $590 \mathrm{~nm}$ (corresponding to the growth of the fully equilibrated species), are not parallel. Both of them yield higher activation energies of 2.7 and $3.2 \mathrm{kca} / \mathrm{mol}$ respectively. The differences are small, but considering the good quality of data (except for some scatter in the low temperature TBAF set), we believe that they are meaningful. They suggest that the original complex consisting of the charge separated species of p-ANTP and the ion pair of the salt undergoes a rearrangement before the final equilibrium is reached. It is reasonable that the reorganization involving the small $\mathrm{F}^{-}$ion gives rise to a higher overall activation energy. In order to extract the rate of this second step and the corresponding $E_{a}$ we will re-fit the 700 and $590 \mathrm{~nm}$ data with a double exponential, in which one component will be determined from the $990 \mathrm{~nm}$ data. This work is in progress.
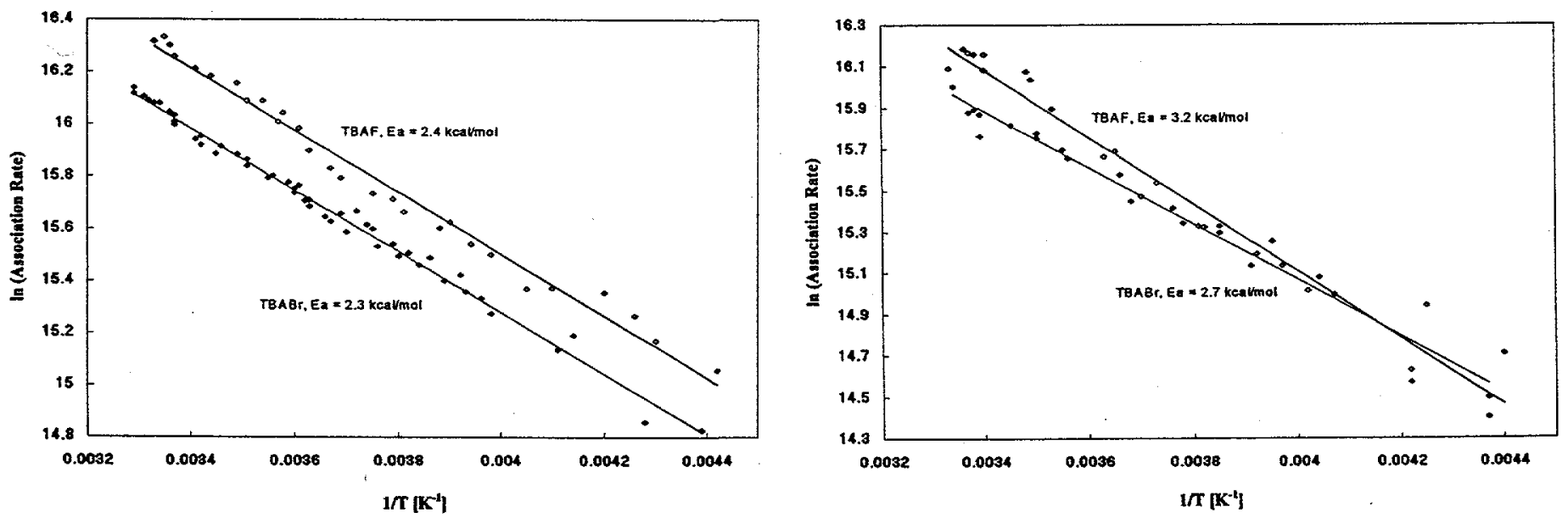

Figure 8. a) Arrhenius plots for ion association rates determined at $990 \mathrm{~nm}$ (1 mM salt concentration); b) Arrhenius plots for ion association rates determined at 710 and $590 \mathrm{~nm}$ (1 mM salt concentration).

To summarize, a 2-step mechanism of the association process can be proposed: 1) initial formation of a complex through a diffusion controlled encounter between the charge separated species and an ion pair of the salt; 2) subsequent rearrangement of the complex, e.g. through loss of one of the ions of the salt. We intend to perform transient Raman experiments in order to test the postulated mechanism. The full length manuscript reporting the results of the variable temperature and ion size dependence work on ion association is in preparation. 
Non-polar solvents: We have recently extended this work to essentially non-polar media and we are probing the ion association dynamics in toluene and benzene. There are very few salts soluble in these solvents: tetrabutylammonium tetrafluoroboron $\left(\mathrm{TBABF}_{4}\right)$, tetrabutylammonium haxafluoroantimonate $\left(\mathrm{TBASbF}_{6}\right)$ and tetrabutylammonium tetrabutylboron (TBATBB). The last one is of limited use since it quickly decomposes in the presence of charge transfer excited states. The maximum concentration of $\mathrm{TBABF}_{4}$ and $\mathrm{TBASbF}_{6}$ in toluene is approximately $3 \times 10^{-3} \mathrm{M}$, and clearly these systems could not be studied using standard fluorescent probes.

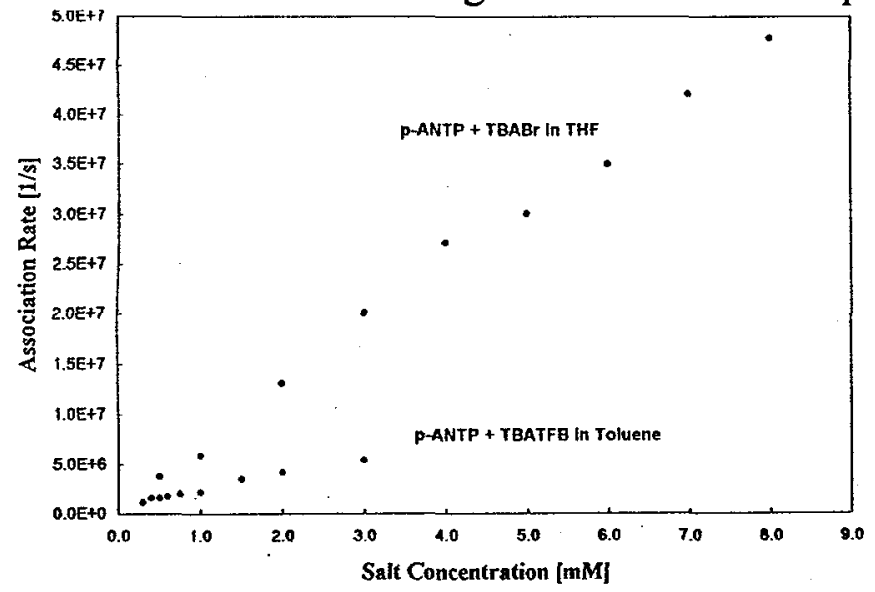

Figure 9. Salt concentration dependence of the ion association rate in $\mathrm{THF}$ and in toluene.

Interestingly, the shifts of the triplet absorption of p-ANTP observed in toluene are not much larger than in THF, contrary to the expectation that the $\sim 3$-times lower dielectric constant of the solvent should be translated into much stronger stabilization of the charge separated species. The preliminary data suggest that the reason for this behavior is the aggregation of the ions and ion pairs of the salt into large clusters before they encounter the probe molecule. This is supported by comparing the salt concentration dependence of the association rate in THF and in toluene (Fig. 9). In THF the dependence was linear, and the pseudo-unimolecular rate of $7.7 \times 10^{9}$ $\mathrm{s}^{-1}$ per 1 mole of salt was close to the diffusion controlled limit. In toluene the rates are considerably slower (even though the viscosity of toluene is only $\sim 1.2$ times higher than that of THF), and the dependence is highly non-linear. At $1 \mathrm{mM}$ salt concentration the pseudounimolecular association rate is $\sim 2.2 \times 10^{9} \mathrm{~s}^{-1}$ per 1 mole of salt. At $3 \mathrm{mM}$ salt concentration it drops further to $\sim 1.7 \times 10^{9} \mathrm{~s}^{-1}$ per 1 mole of salt, indicating that the clusters grow in size with the increasing salt concentration. Further investigation of the aggregation effects in non-polar media, including their temperature dependence, is in progress. 
Intramolecular ion association: The work on systems capable of intramolecular ion association upon photoexcitation has advanced significantly. Rigidly linked donor-acceptor assemblies with a permanent ion tethered to the molecule via variable length polymethylene chains were prepared. The dynamics of intramolecular ion association will be studied as a function of the chain length, medium viscosity and polarity, as well as temperature.<smiles>CC1c2cc(N)ccc2-c2ccc([N+](=O)[O-])cc21</smiles>

The compounds were prepared in five steps from fluorene:

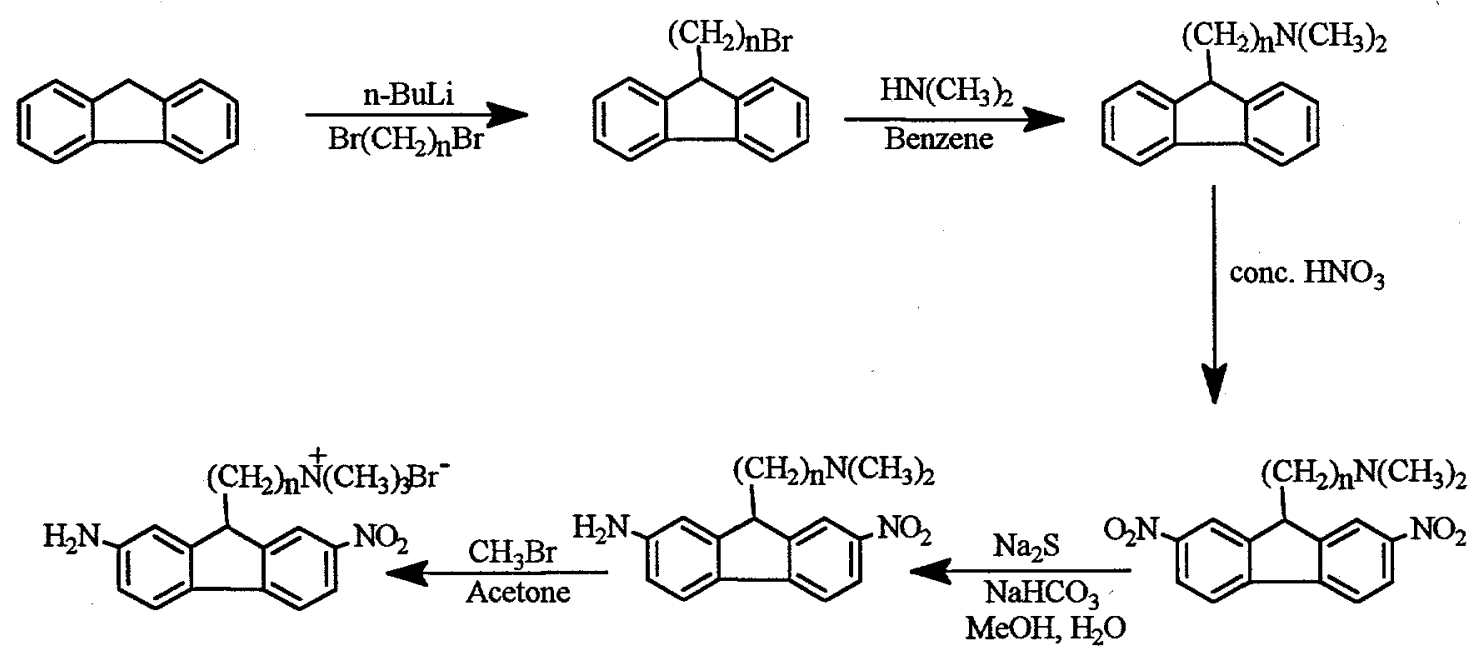

Fluorene was alkylated with 1,n-dibromo-n-ane (n-butyllithium as the base). A mixture, with the 9-(1-bromo-n-yl)-fluorene as the major product, was obtained. Purification of all $(n=3-10)$ alkylfluorenes was achieved by chromatography on silica-gel with iso-octane as eluent. We decided against using n-bromo-N,N-dimethyl-n-yl-amine for alkylation was that we could separate easily mixture after alkylation with dihalides.

The amination of the chain with dimethylamine was achieved in dry benzene. The reaction was complete after overnight heating. Purification was not necessary because only one product was formed. The equilibrium is favorable because dimethylammonium bromide precipitates in 
benzene. The isolation required only isolation and removing benzene. Pure product was obtained in yield of more than $90 \%$.

The N,N-dimethyl-n-(9-(fluorenyl)-ylamine was treated with concentrated $\mathrm{HNO}_{3}$ at room temperature yielding the 2,7-dinitro product. The products were isolated as pure yellow salts.

Reduction of the N,N-dimethyl-n-(9-(2,7-dinitrofluorenyl)-ylamine was carried out in the usual way with $\mathrm{Na}_{2} \mathrm{~S} \cdot 9 \mathrm{H}_{2} \mathrm{O}$.

The final product, N,N-dimethyl-n-(9-(2-amino-7-dinitrofluorenyl)-yl-ammonium bromide is extremely hygroscopic. It requires work under nitrogen atmosphere and rigorously dry solvents (ether and acetone). The yield was over $80 \%$ and no further purification was necessery except for drying.

A set of 2-amino-7-nitro-9-n-yl-fluorene reference compounds with methyl terminated tethers, which are not capable of intramolecular ion association, was also prepared:<smiles>CCC1c2cc(N)ccc2-c2ccc([N+](=O)[O-])cc21</smiles>

The 2-amino-7-nitro-9-n-yl-fluorenes were prepared in three steps from fluorene:
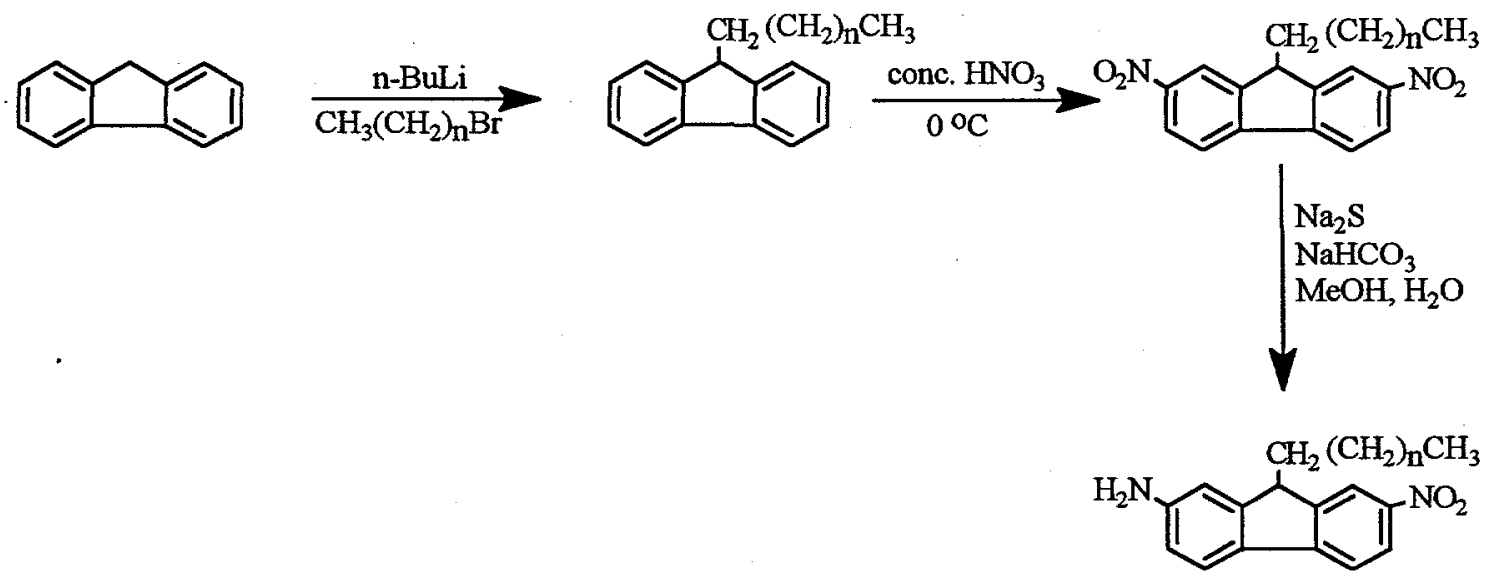

The alkylation and reduction were carried out in the same way as described above. Nitration was performed at $0{ }^{\circ} \mathrm{C}$ and mixture of three products was obtained: mono-, di- and trinitro. The mixture was separated by chromatography on silica-gel with a $1: 4$ mixture of $\mathrm{CH}_{2} \mathrm{Cl}_{2}$ and hexane.

The spectroscopic characterization of the model compounds with tethered ions is in progress. 


\section{The role of MO and vibrational mode symmetry in intramolecular excitation transfer}

Another area of our interest is the role of MO and vibrational mode symmetry in determining the electronic coupling between the donor and the acceptor. We have been investigating the symmetry dependence of the electronic coupling and singlet excitation transfer in a series of bifluorenes. All reported experiments were performed in a supersonic molecular beam in collaboration with Dr. Don Levy. The detailed discussion of the findings is provided in the attached manuscript entitled "Vibronic Coupling and Energy Transfer in Bichromophoric Molecules: The Effect of Symmetry". Therefore, we will discuss only the most fundamental results obtained on spirobifluorene (SBF) and its analogue, $d_{g} h_{g}-\mathrm{SBF}$, in which one chromophore has been perdeuterated. Spirobifluorenes (Fig. 1) are unusual because symmetry considerations and MO calculations predict total cancellation of both Coulomb and exchange integrals between the local LUMO orbitals, and hence also between the localized $S_{1}$ states in these $D_{2 d}$ molecules.

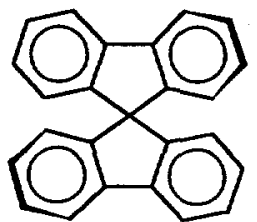

spirobiflucrene (SBF)

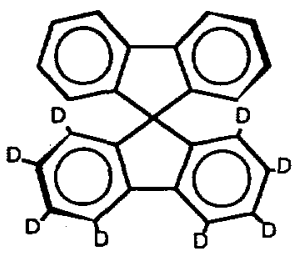

$d_{8} h_{6}-$ spirabilituorene $\left(d_{8} h_{8}-S B F\right)$

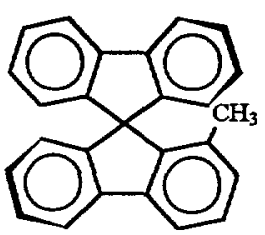

1-methylspirobifiuorene (1M-SBF)

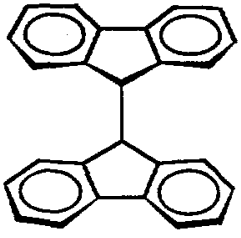

9,9-bifluorene (BF)

Figure 1. The investigated bifluorenes.

Indeed, as predicted by symmetry, no exciton splitting was detected in the excitation spectrum of SBF and $d_{8} h_{8}-\mathrm{SBF}$ in supersonic jet experiments with resolution of $0.05 \mathrm{~cm}^{-1}$. 9 Only one origin transition red-shifted by $738 \mathrm{~cm}^{-1}$ with respect to monomeric fluorene is observed in SBF (Fig. 2). In the $d_{s} h_{8}$-SBF two origin transitions are observed (Fig. 2.). However, these arise due to the difference in the zero point energies of the $d_{8^{-}}$and $h_{8^{-}}$fragments, rather than due to exciton splitting. The difference between the two origins of $d_{8} h_{8}$-SBF is well reproduced by the difference between the origin of fluorene and deuterated fluorene (Fig. 2). The localized $S_{1}$ states of the two fluorene units in spirobifluorenes are truly uncoupled from one another. Only in the bifluorene (BF) with non-orthogonal chromophores an exciton splitting of $57 \mathrm{~cm}^{-1}$ was observed. $^{10}$ 


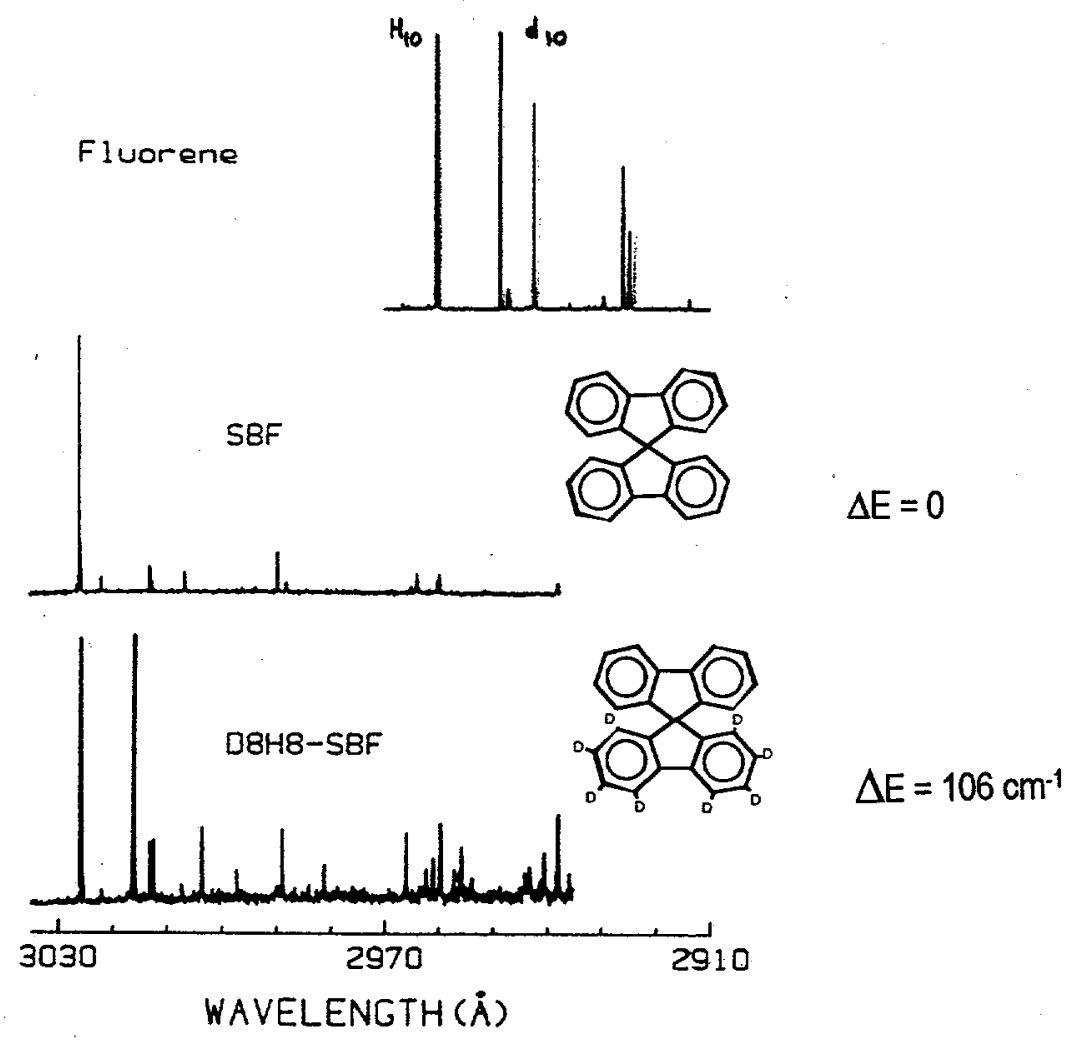

Figure 2. Fluorescence excitation spectra of fluorene, $d_{10}$-fluorene, BF, SBF and $d_{8} h_{8}-S B F$.

Even when the electronic coupling between the localized states is zero in the zero-point vibrational levels, the coupling may be turned on if the system is promoted to a vibrationally excited state of appropriate symmetry. This effect is a strict analogue of the Herzberg-Teller coupling ${ }^{11}$ which through a vibrational distortion allows electronic absorption in molecules with symmetry forbidden transitions. In the case of electronic energy transfer in donor-acceptor systems the relevant electronic states are localized on two different chromophores and become coupled by non-totally symmetric interchromophore vibrations.

This effect can be probed by exciting a bichromophoric molecule in to a vibrationally excited state of the $S_{1}$ state and monitoring how the excitation is redistributed between the two chromophores. In degenerate systems, like SBF, this can be accomplished by time resolved fluorescence depolarization measurements. ${ }^{12}$ The molecular beam apparatus available to us does not have this capability. This difficulty can be solved if the two chromophores have distinct spectra. Therefore, we have prepared the $d_{8} h_{8}$-SBF in which, as mentioned above, the isotopic substitution introduces a small offset between the energy levels of the chromophores, yet it preserves the essential electronic symmetry of the molecules. The offset of $106 \mathrm{~cm}^{-1}$ between the two vibrational manifolds allows us to: 1) selectively excite a vibronic state localized of a given 
chromophore; 2) detect energy transfer between the chromophores by dispersing the emission and monitoring the partitioning of fluorescence.

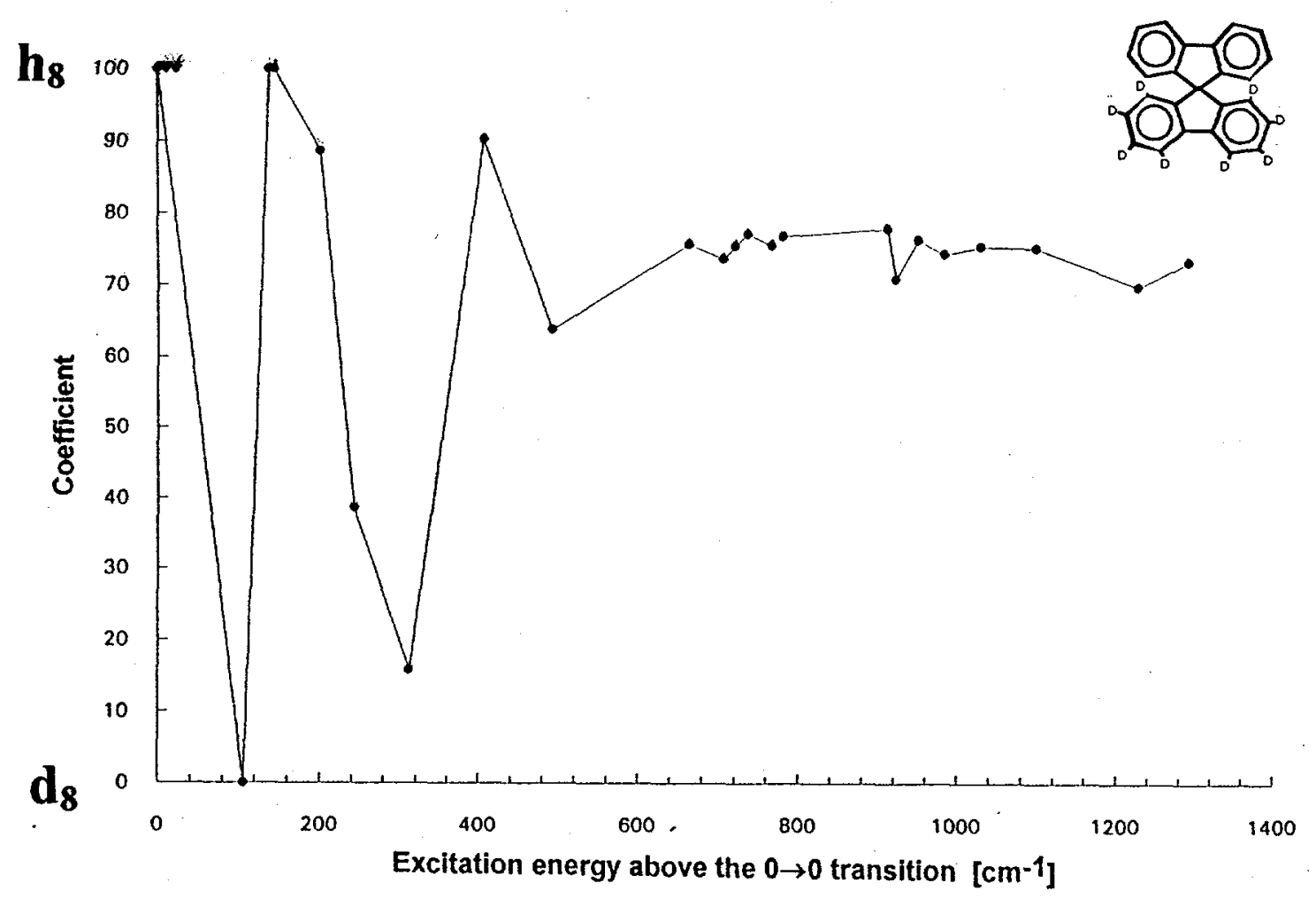

Figure 3. Partitioning of the emission between the $d_{8}$ - and $h_{8}$ - chromophores as a function of the vibrational excitation of the $S_{1}$ state of $d_{8} h_{8}-S B F$.

The complete dispersed emission fluorescence spectra of $d_{8} h_{8}$-SBF excited into the first 22 vibrationally excited states of the $S_{1}$ state of either chromophore are included in the attached manuscript. ${ }^{13}$ Here we summarize these results in Figure 3, which shows the percentage of emission from either the $d_{\delta^{-}}$or $h_{\delta^{-}}$chromophore as a function of the vibrational excitation above the $0-0$ transition. A very interesting pattern is revealed: Up to approximately $200 \mathrm{~cm}^{-1}$ above the lower origin no excitation transfer is observed. The emission occurs purely either from the $h_{\delta}$ or the $d_{8^{-}}$- half of the molecule, depending on which one has been excited. Excitation of modes $>200$ $\mathrm{cm}^{-1}$ above the origin leads to onset of dual emission, and hence electronic coupling and excitation transfer. The relative intensity of the $d_{8}$ - and $h_{8}$-fluorene bands varies erraticaly depending on the excited vibronic state. At $650-700 \mathrm{~cm}^{-1}$ excess vibrational energy a plateau is reached and the ratio of the two emissions no longer depends on the identity of the excited vibronic state. 

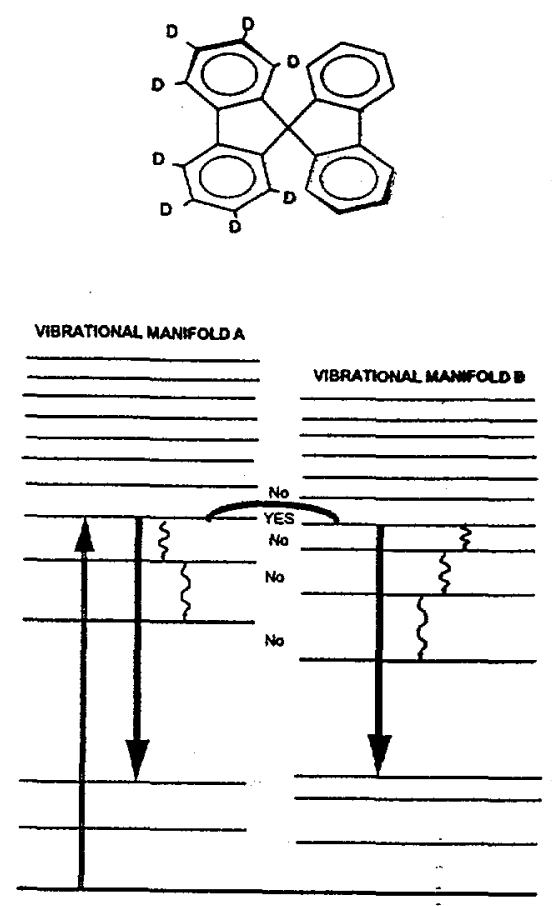

Requirements for coupling and excitation transfer 1) matching or near matching of levets

2) proper symmetry of the vibrational mode.

Figure 4. Schematic representation of the level matching in $d_{8} h_{8}-S B F$.

The observed behavior can be explained as follows: In the lowest portion of the vibrational manifold in a weakly non-degenerate molecule like the $d_{8} h_{8}$-SBF the energy mismatch between the two halves makes electronic excitation transfer impossible. The levels are sparse in this region and the coupling is truly symmetry forbidden. As the excitation energy increases so does the density of states on both sides. Incidental degeneracies or near-degeneracies of the opposing states of appropriate symmetry will occur and they will lead to state mixing and energy transfer. This is the "small molecule" limit of the radiationless transition theory. At even higher excitation energies the density of states becomes so high that the random degeneracies are no longer important, as there is always a nearly isoenergetic state of appropriate symmetry available. This region of statistical mixing corresponds to the "large molecule" limit of the radiationless transition theory. In this regime electronic energy transfer and IVR occur at a similar time scale.

To our knowledge, this is the first example of the transition from the "small molecule" regime to the "large molecule" regime observed in such large bichromophoric system. The described experiments demonstrate with clarity that the electronic coupling and excitation transfer can be completely switched off by MO symmetry. However, when the density of states becomes sufficiently high, the often invoked simple notion of symmetry forbidden donor-acceptor electronic coupling is not fully appropriate. 
Intramolecular dispersive interactions: The intramolecular dispersive interactions which are always present in bichromophoric molecules were also investigated. The polarizable electron distribution of one chromophore responds to the transition dipole localized on the other chromophore. These "self-solvation" effects do not lead to energy transfer, however, they do contribute to the diagonal matrix elements and hence lead to spectral shifts. It is important to be able to distinguish between shifts arising due to the dispersive effects and these arising due to the true exciton splittings. We have prepared a series of model reference compounds with a large energy gap between the $S_{1}$ state of the fluorene chromophore and the $S_{1}$ state of the other chromophores (one or more phenyl groups in various arrangements). As a result of this large $\sim 3500 \mathrm{~cm}^{-1}$ gap the resonant coupling with the fluorene $S_{1}$ state is insignificant, and the entire spectral shift of the fluorene emission should occur due to dispersive interactions. The reference compounds were selected in such a manner that the molecular volume and chemical attachment of the polarizable moieties attempts to mimic the volume and arrangement of the corresponding fluorene chromophores. The red shifts of the fluorescence origins were measured under the same supersonic expansion conditions.

The reference compounds accounted reasonably well (to within $\sim 15 \%$ ) for the red shifts observed in bifluorenes. Since the polarizability tensor is anisotropic, the orientation of the phenyl groups with respect to the fluorene transition dipole is important in determining the magnitude of the interaction and it is unrealistic to expect an exact agreement just on the basis of molecular volume.

For more details about this work, please, see the attached manuscript entitled "Vibronic Coupling and Energy Transfer in Bichromophoric Molecules: The Effect of Symmetry". The continuation of this project, focusing on the role of incidental degeneracies of the energy levels of the two chromophores and the density of states, is described in the Renewal Proposal. 


\section{Zwitter-ionic and biradicaloid excited states of twisted olefins}

Our recent entry into this field has been prompted primarily by the new project on ET transition metal complexes with diazobifluorenylidene and tetraazobifluorenylidene twisted olefin ligands (see the next chapter), and the need for better understanding of the photophysics of these systems. The parent hydrocarbons were selected for the initial study because their spectroscopic behavior is expected to be simpler than that of their azo-analogues.

Photophysics of bifluorenylidene and biphenathrenylidene: Both $\mathrm{x}$-ray diffraction data and MO calculations indicate that because of the steric interaction between the opposing aromatic rings the molecules of 9,9'-bifluorenylidene and bi- $4 H$-cyclopenta[def]phenathren-4-ylidene (which will be referred to as biphenanthrenylidene). are twisted about the central double bond by $39^{\circ}$ and $31^{\circ}$, respectively. ${ }^{14}$

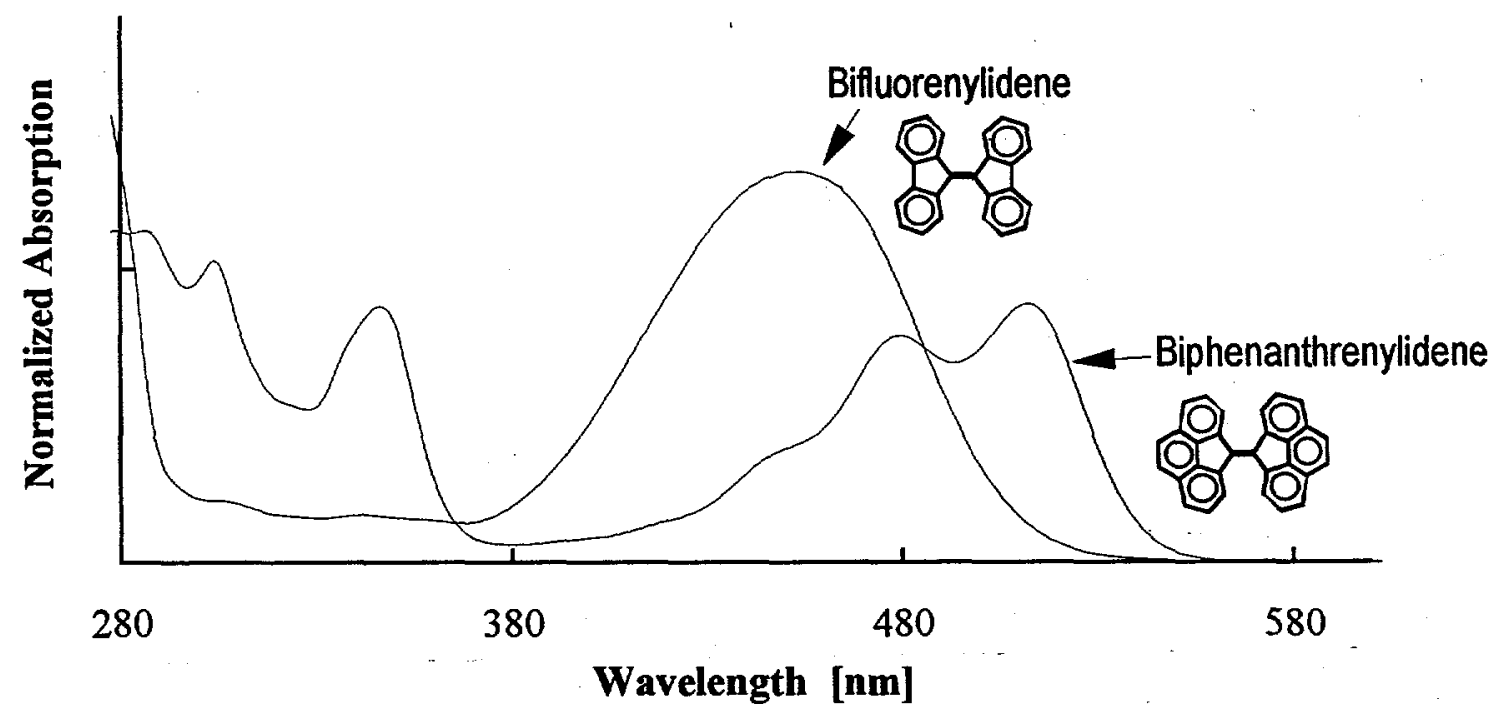

Figure 1. UV-Vis absorption spectra of bifluorenylidene and biphenathrenylidene.

As a result of this large ground state distortion, the UV-Vis absorption spectra of bifluorenylidene and biphenathrenylidene are composed of long wavelength bands corresponding to delocalized transitions, as well as bands at short wavelengths corresponding to essentially localized transitions. The visible portion of the spectrum of bifluorenylidene consists of a very strong featureless band, with $\lambda_{\max }=454 \mathrm{~nm}$ and $\varepsilon=26700$. The corresponding long wavelength band of biphenanthrenylidene, $\lambda_{\max }=512 \mathrm{~nm}$, exhibits more vibrational structure, and has a considerably lower extinction coefficient, $\varepsilon=8800$. Both compounds are bright red. 
Bifluorenylidene and biphenanthrenylidene can be viewed as special members of the stilbene family. The rigidity of the chromophores in these molecules limits the evolution on the excited state surface strictly to the rotation around the central double bond. Upon excitation molecules of stilbene type are known to relax to a singlet state with $\mathrm{a}>\mathrm{C}=\mathrm{C}<\mathrm{twist}$ angle of $90^{\circ}$, and intersystem cross to a triplet state with the same dihedral angle of $90^{\circ}$ (due to very rapid nonradiative decay to the ground state the triplet yield is usually very low). ${ }^{15}$

Bifluorenylidene exhibits very rapid non-radiative decay from the singlet state and the triplet state. As a result, no trace of transient absorption could be detected at nanosecond timescale either with direct excitation, or by sensitization with triplet benzophenone.

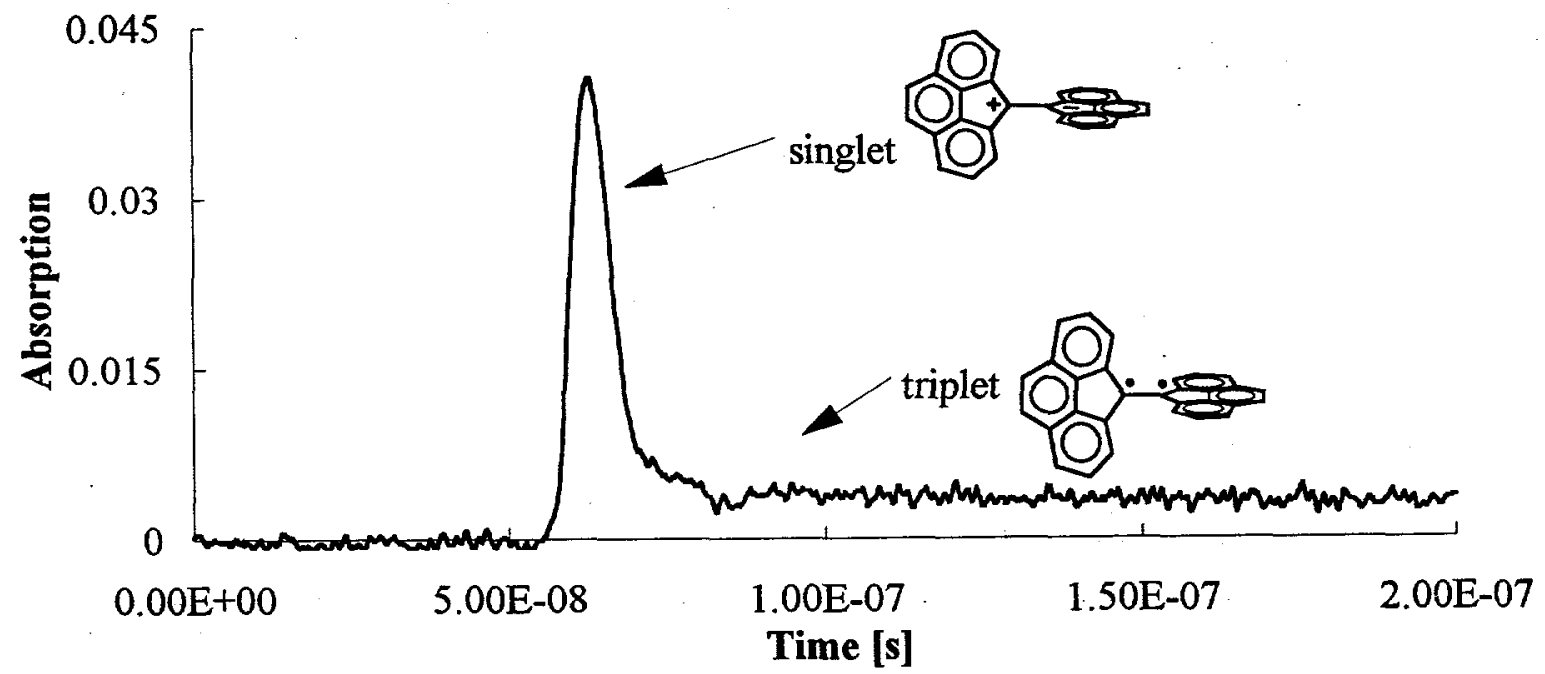

Figure 2. Transient absorption profile of $0.2 \mathrm{mM}$ biphenanthrenylidene in benzene, excitation at $532 \mathrm{~nm}$, pulse width $7 \mathrm{~ns}$.

In contrast, in biphenanthrenylidene both the excited singlet and triplet states are long lived and their absorption can be easily monitored (Fig. 2). The transients consist of a fast component $\tau<2 \mathrm{~ns}$, which closely follows the profile of the laser pulse, and a weaker much slower component, $\tau \approx 5 \mu$ s. Identical transients are obtained upon $532 \mathrm{~nm}$ excitation of the delocalized visible band, and $355 \mathrm{~nm}$ excitation of the essentially localized UV band.

Neither the singlet nor the triplet absorption of biphenathrenylidene could be detected in a low temperature glass (3-methylpentane below $-100{ }^{\circ} \mathrm{C}$ ). We attribute the disappearance of the transient to the rigidity of the frozen medium which precludes the relaxation of the molecule's geometry to the $>\mathrm{C}=\mathrm{C}<$ dihedral angle of $90^{\circ}$. When the measurement was performed in a low temperature liquid, spectra identical to the room temperature ones were obtained. 


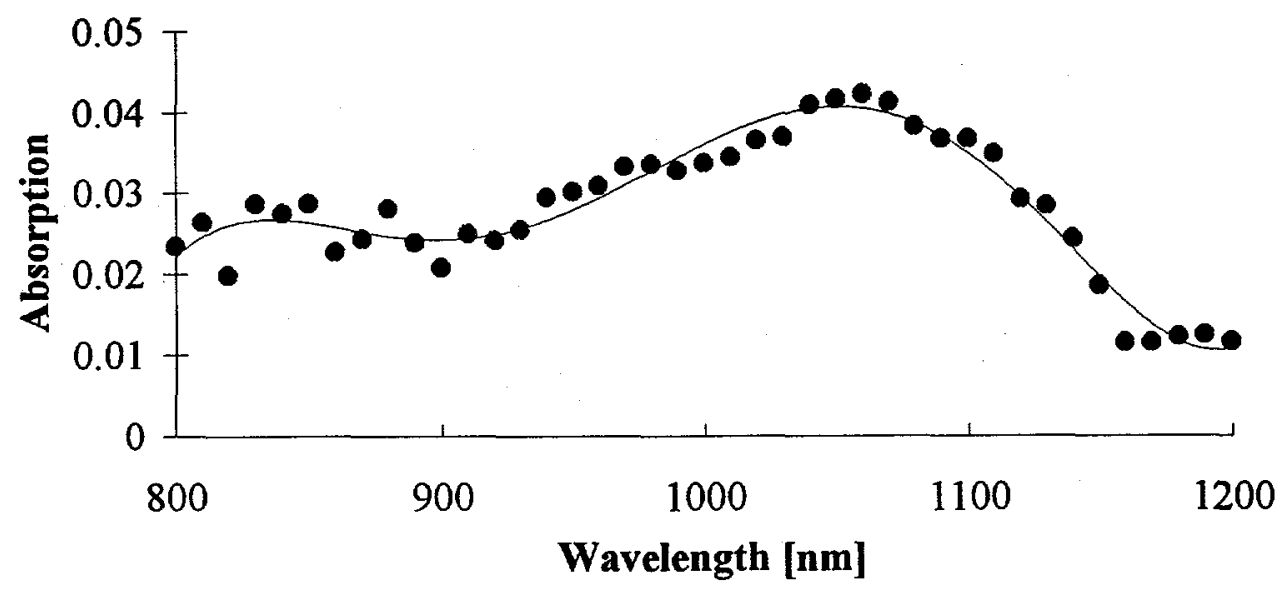

Figure 3. Excited singlet state absorption of $0.2 \mathrm{mM}$ biphenanthrenylidene in benzene, excitation at $355 \mathrm{~nm}$, pulse width $5 \mathrm{~ns}$.

The spectrum of the singlet component extends into near IR and peaks at approximately $1050 \mathrm{~nm}$ (Fig. 3). It resembles a superposition of the spectra of the radical cation and the radical anion of phenanthrene. ${ }^{16}$ Therefore, we assign it to the $90^{\circ}$-twisted zwitter-ionic singlet state of II.
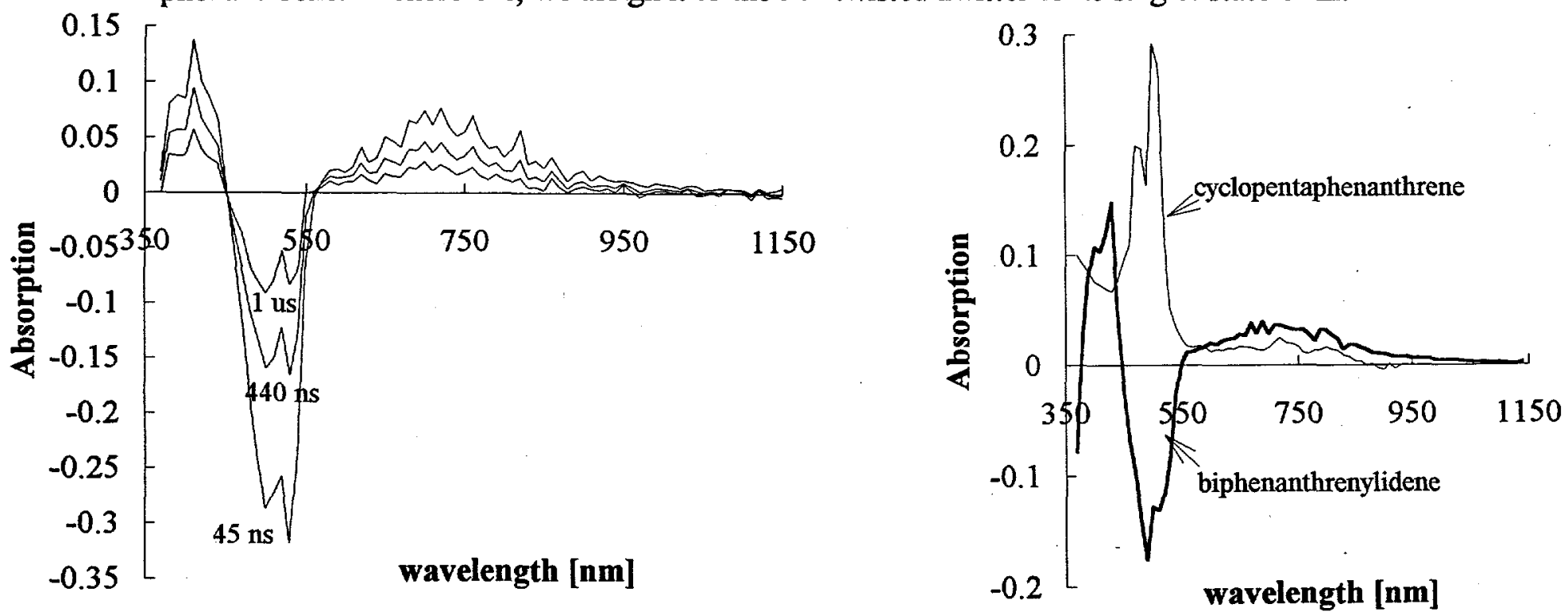

Figure 4. a) Time evolution of the transient triplet absorption spectrum of $0.2 \mathrm{mM}$ II in benzene, direct excitation; b) sensitized T-T absorption spectrum of biphenathrenylidene. The T-T spectrum of monomeric cyclopentaphenathrene is included as a reference.

The triplet spectrum of biphenanthrenylidene can be obtained either by direct excitation or by sensitization, e.g. with benzophenone (Fig. 4). The spectrum is dominated by a prominent bleach of the visible band of the ground state absorption. The bleach allows us to estimate the triplet yield, which is surprisingly large, $\varphi_{\mathrm{T}} \approx 0.3$. The broad $\mathrm{T}_{\mathrm{n}} \leftarrow \mathrm{T}_{1}$ absorption peaks at $\sim 710 \mathrm{~nm}$ and is clearly very different from the spectrum of the fast component (Fig. 3). We tentatively assign it to the biradicaloid twisted triplet state of biphenanthrenylidene. 
The reasons for the dramatically different excited state behavior of bifluorenylidene and biphenanthrenylidene are not clear yet. A simple zero-order analysis of the energetics of the two molecules is not very helpful in this regard: The combined difference of the IPs and the electron affinities of monomeric fluorene and phenanthrene (no data are available for the more relevant cyclopentaphenathrene) is approximately $+0.2 \mathrm{eV}$ (i.e. it should take $\sim 0.2 \mathrm{eV}$ more to create the zwitter-ionic state of bifluorenylidene than that of biphenanthrenylidene). The difference between the $T_{1}$ states of fluorene and phenanthrene is also only $+0.2 \mathrm{eV}$. These values are closely paralleled by the difference in the energy of the lowest transitions in in these compounds, which on the basis of the $\lambda_{\max }$ values from Fig. 1 is approximately $0.3 \mathrm{eV}$. Therefore, it is not obvious why: 1) the zwitter-ionic singlet state of biphenanthrenylidene is so long lived in comparison with bifluorenylidene; 2) why the ISC to the twisted triplet state is so much more efficient in biphenanthrenylidene than in bifluorenylidene.

We are addressing these questions through higher time resolution measurements and through computations.

The mechanism of photoisomerization of tetraphenylethylene (TPE): This contribution has been stimulated by the recent experimental paper by Waldeck and Zimmt ${ }^{17}$. The full discussion of our findings is described in the attached paper and, therefore, the only the most important findings are summarized below: Despite its apparent higher symmetry TPE is a more complex molecule than stilbene, and it displays considerably more complex photophysics. Three distinct conformational minima were found on the excited state surface of TPE (AM1 and UHF/3$21 \mathrm{G}^{*}$ ), in contrast with one minimum of silbene. This fact has been overlooked in all experimental studies of TPE. The existence of the minima is determined primarily by simple steric interactions of the crowded phenyl rings, rather than by subtle electronic effects. Two of the local minima (which are mirror images of one another) maintain the $\mathrm{D}_{2}$ symmetry of the ground state, and we believe that they correspond to the "relaxed" emissive state of TPE. The true minimum, at central $>\mathrm{C}-\mathrm{C}<$ bond twist angle of $90^{\circ}$, is of $\mathrm{C}_{2 \mathrm{~h}}$ symmetry, and it corresponds to the nonemissive twisted zwitter-ionic state of TPE. The existence of the excited state conformers is consistent with the much long lifetime of the zwitter-ionic state of TPE (1.5 ns) in comparison 
with stilbene ( $200 \mathrm{fs})$. This difference of 4 orders of magnitude can be easily rationalized in terms of conformational trapping, which is possible in TPE, but not in stilbene.

AM1 exploration of the isomerization reaction coordinate shows that the lowest energy path for interconversion between the conformers proceeds through a very interesting correlated "gearing" rotation of the four phenyl groups of TPE, in agreement with the "ring flip" mechanism studied by Mislow, Gust and Finochiaro for ground state molecules. ${ }^{18}$ On the basis of these considerations we have postulated the most probable sequence of events in the photoisomerization of TPE.

We believe that the finding of conformational local minima on the excited state surface of TPE is a very important addition to the continuing discussion of the photophysics of stilbene-like molecules. The existence of such minima is not intuitively obvious, and most likely for that reason it has not been recognized by other investigators. For more details, please, see the attached manuscript "On the Photoisomerization Coordinate of Tetraphenylethylene".

\section{New Electron Transfer Transition Metal Complexes}

The general synthetic schemes developed during the preparation of spirobifluorenes and twisted olefins (see the previous chapters) have been utilized to synthesize several new ligands for electron transfer transition metal complexes. ${ }^{19}$ Three of them are terminal diazo- ligands (Fig. 1), and the other three are bridging tetrazo- ligands (Fig. 2). The synthesis of all of them, except for the most difficult tetraazospirobifluorene IV, has been completed. All ligands consist of two chromophores, either fluorene and 4,5-diazofluorene, or two 4,5-diazofluorenes, linked at the 9carbon atom. The ligands with the spiro- or $\sigma$-bond linkage are colorless. The ligands with the double bond linkage are orange/red.
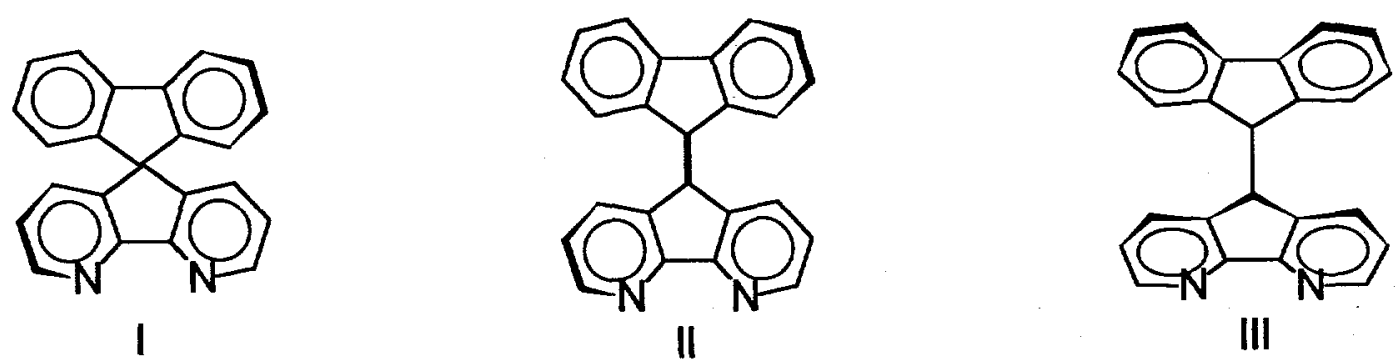

Figure 1. Terminal ligands: 4,5-diazospirobifluorene I, 4,5-diazobifluorenylidene II and 4,5-diazobifluorene III. 


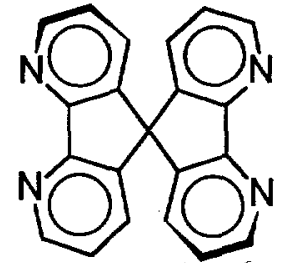

IV

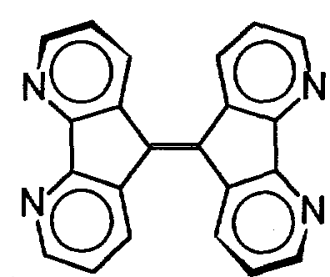

$\checkmark$

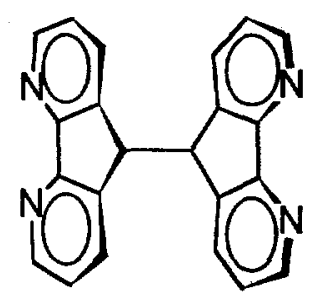

$\mathrm{VI}$

Figure 2. Bridging ligands: 4,4',5,5'-tetraazospirobifluorene IV, 4,4',5,5'-tetraazobifluorenylidene $V$, and 4,4',5,5'-tetraazobifluorene VI.

The new ligands $\mathbf{I}, \mathbf{I I}$ and $\mathbf{I V}, \mathbf{V}$ posses high structural rigidity and interesting electronic properties (see the previous chapters on the isoelectronic spirobifluorenes and twisted olefins). In spiranes I and IV the localized LUMOs are effectively decoupled, however, the corresponding HOMOs are relatively strongly coupled because of their different symmetry. Because of the sterically induced $>30^{\circ}$ twist of the central double bond in ligands $I I$ and $V$ the $\pi$-systems of these molecules are not fully delocalized. This leads to the presence of essentially delocalized long wavelength transitions, as well as short wavelength "monomer"-like transitions in the UV-Vis spectra. ${ }^{19}$ We will compare the electronic properties of binuclear complexes with the weakly coupling bridge IV and the strongly coupling bridge $\mathrm{V}$.

We have already prepared crystalline mononuclear $\mathrm{Ru}(\mathrm{III})$ complex of the 4,5diazospirobifluorene I. Its x-ray structure has been recently solved. It reveals the presence of intricate $7 \times 9 \AA$ zeolite-like channels filled with solvent molecules. This structure explains the unexpectedly low density of this material $\left(\sim 1.0 \mathrm{~g} / \mathrm{cm}^{3}\right)$. Spectroscopic characterization of this complex is in progress.
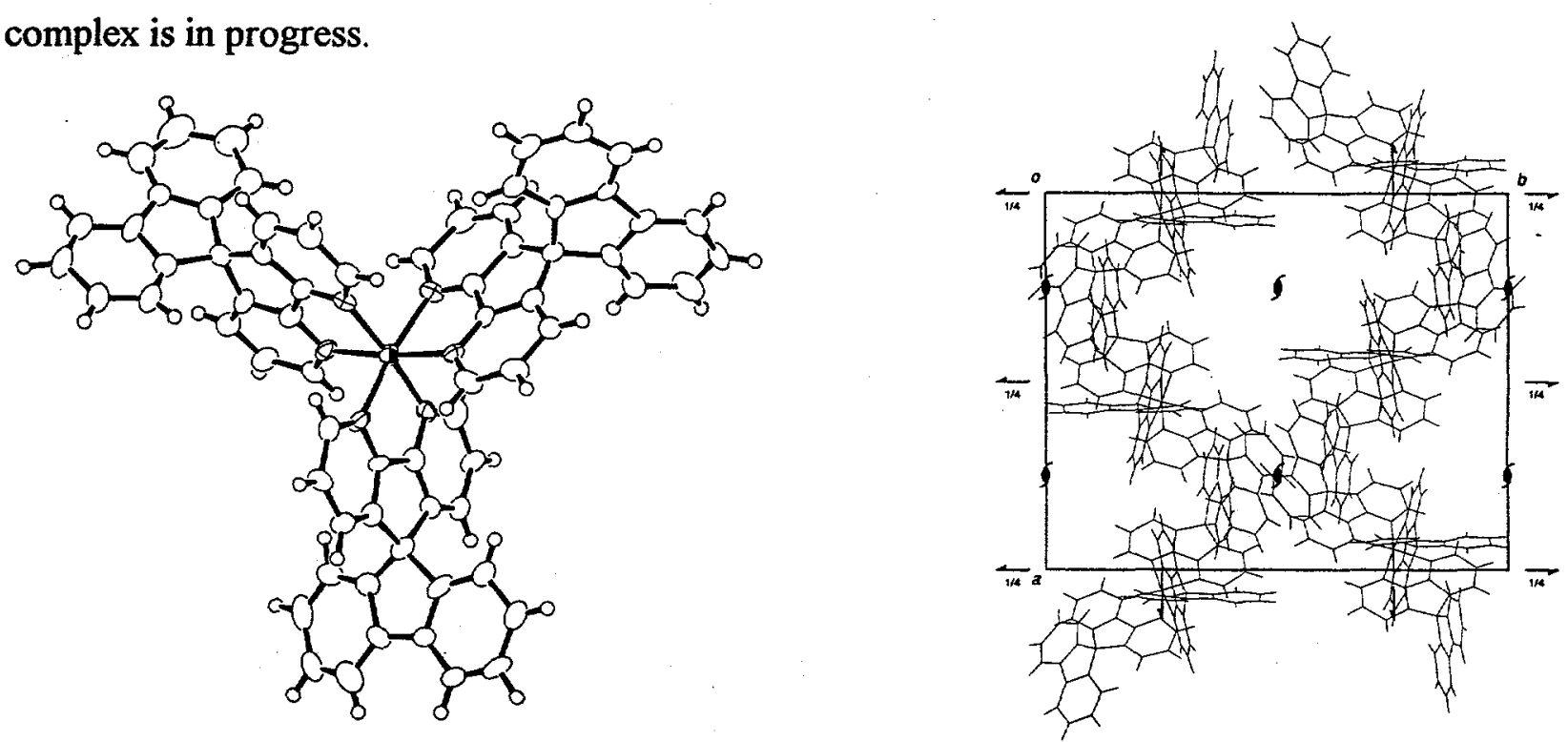

Figure 3. The $x$-ray structure and a fragment of the three-dimensional network of the tris(diazospirobifluorene)Ru(III) chloride. 


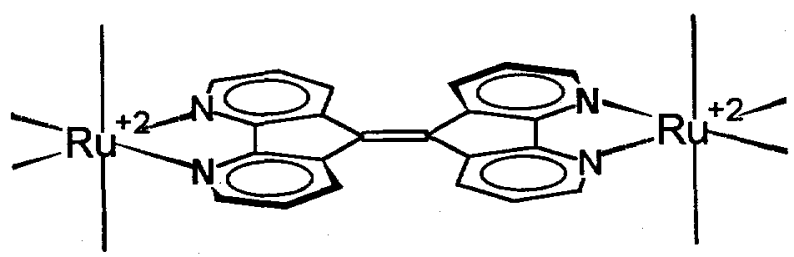

Figure 4. The binuclear complex [bis(bipirydyl) $\left.\mathrm{Ru}^{2+}\right]-4,5,4^{\prime}, 5^{\prime}$-tetraazobilfuorenylidene[bis(bipirydyl) $\mathrm{Ru}^{+2}$ ].

The crystals of our first binuclear complex, the $\left[\right.$ bis(bipirydyl) $\left.\mathrm{Ru}^{2+}\right]-4,5,4^{\prime}, 5^{\prime}-$ tetraazobifluorenylidene-[bis(bipirydyl) $\left.\mathrm{Ru}^{+2}\right]$ chloride are currently being grown.

The long term objective of this project is the preparation and spectroscopic, as well as electrochemical, investigation of extended 3-dimensional organometallic networks built from the above ligands and transition metal ions.

\section{Related efforts}

In May of this year the PI submitted an NSF instrumentation proposal requesting funding for a $\$ 258,000$ tunable pico/femtosecond regeneratively amplified $\mathrm{Ti}: \mathrm{Al}_{2} \mathrm{O}_{3}$ laser system. The coinvestigators are Russell Schmehl and Vaidyanathan Ramamurthy, both of Tulane University. If funded, the laser will be housed and operated by the PI's group. It will be used extensively in the PI's and Russell Schmehl's Department of Energy funded research.

\section{Summary}

We have described the current stage of our Department of Energy sponsored research. The most important accomplishments include the detailed study of the MO symmetry effects on intramolecular excitation transfer in bichromophores, and the demonstration that the dynamic shifts of transient charge transfer absorption bands can be used to probe the dynamics of and energetics ion association processes in a very broad variety of media.

We would like to point out that up to December of 1992 our research group consisted of the PI and one undergraduate student. Since then the group expanded and includes two 3rd year graduate students, one 1st year graduate student, and a postdoctoral associate. Therefore, a corresponding increase in productivity can be expected in the next funding cycle. 


\section{References:}

1. P. Piotrowiak, J. R. Miller, J. Phys. Chem. 97, 13052 (1993).

2. D. M. Tiede, D. K. Hanson, in The Photosynthetic Bacterial Reaction Center II (J. Breton, A. Vermeglio, eds.) Plenum Press, New York, p. 341-350 (1992).

3. a) D. Huppert, V. Ittah, E. M. Kosower, Chem. Phys. Lett. 159, 267 (1989); b) C. F. Chapman, M. Maroncelli, J. Phys. Chem. 95, 9095 (1991).

4. D. B. O'Connor, G. W. Scott, K. Tran, D. R. Coulter, V. M. Miskowski, A. E. Stiegman, G. E. Wnek, J. Chem. Phys. 97, 4018 (1992).

5. P. Piotrowiak, R. Kobetic, T. Schatz, G. Strati, J. Phys. Chem. 99, 2250 (1995).

6. M. Szwarc, Ions and Ion Pairs in Organic Reactions, Wiley-Interscience, New York (1972).

7. F. Kohlrausch, Z. Elektrochem. 13, 333, (1907).

8. E. Bart, D. Huppert, Chem. Phys. Lett. 195, 37 (1992)

9. N. A. van Dantzig, D. H. Levy, C. Vigo, P. Piotrowiak, J. Chem. Phys. accepted for the September 22, 1995 issue.

10. P. G. Smith, S. Gnanakaran, A. J. Kaziska, S. M. Hong, R. M. Hochstrasser, M. R. Topp, J. Chem. Phys. 100, 3384 (1994).

11. G. Herzberg, E. Teller, Z. Phys. Chem. B 21, 410 (1931).

12. a) Y. R. Kim, P. Share, M. Pereira, M. Sarisky, R. M. Hochstrasser, J. Chem. Phys. 91, 7557 (1989); b) T. Troxler, B. A. Prior, M. R. Topp, Chem. Phys. Lett. 239, 44 (1995).

13. Table 2, p. 18-19, Figures 6-9, in reference 9 .

14. F. H. Herbstein, Acta. Cryst. B 47, 288 (1991).

15. D. H. Waldeck, Chem. Rev. 91, 415 (1991) and references therein.

16. T. Shida, Electronic Absorption Spectra of Radical Ions, Elsevier, Amsterdam, The Netherlands, (1988).

17. J. Ma, G. B. Dutt, D. H. Waldeck, M. B. Zimmt, J. Am. Chem. Soc. 116, 10619 (1994).

18. a) D. Gust, K. Mislow, J. Am. Chem. Soc. 95, 1535 (1973); b) P. Finochiaro, D. Gust, K. Mislow, J. Am. Chem. Soc. 98, 4952 (1974).

19. P. Piotrowiak, R. Kobetic, T. Schatz, S. Tapper, Bull. Pol. Acad. Sci. 42, 441 (1994). 
Publications resulting from the DOE supported research, 1993-1995:

Electrolyte Effects in Intramolecular Electron Transfer, P. Piotrowiak, ACS Advances in Chemistry, invited paper, in preparation.

Long-Lived Excited States of a Twisted Olefin, P. Piotrowiak, G. Strati, in preparation for J. Am. Chem. Soc.

Ion Association with a Photoinduced Charge Separated Species: Ion Size and Temperature Dependence, P. Piotrowiak, T. Schatz, R. Kobetic, in preparation for J. Am. Chem. Soc.

On the Photoisomerization Coordinate of Tetraphenylethylene, P. Piotrowiak, Chem. Phys. Lett., 1995, accepted.

Vibronic Coupling and Energy Transfer in Bichromophoric Molecules: The Effect of Symmetry, N. A. van Dantzig, D. H. Levy, C. Vigo, P. Piotrowiak, J. Chem. Phys., 1995, accepted.

Transient Charge Transfer Absorption Bands as Probes of Ion-Pairing Dynamics and Energetics, P. Piotrowiak, R. Kobetic, T. Schatz, G. Strati, J. Phys. Chem, 1995, 99 , 2250.

Novel Ligands for Electron Transfer Metal Complexes, P. Piotrowiak, R. Kobetic, T. Schatz, S. Tapper, Bull. Pol. Acad. Sci., 1994, 42, 441. Invited paper, memorial issue.

Specific Ion-Pairing Effects in Weakly Exoergic Electron Transfer, P. Piotrowiak, Inorg. Chem. Acta, 1994, 225, 269. Invited paper, special Electron Transfer issue.

Van der Waals Complexes of Bichromophore Spirobifluorene, N. A. van Dantzig, P. Piotrowiak, D. H. Levy, Chem. Phys. Lett., 1994, 223, 127.

Counterion Effects in Intramolecular Charge Transfer in Radical Anions, P. Piotrowiak, J. R. Miller, J. Phys. Chem., 1993, 97, 13052.

\section{Invited talks at universities:}

MO Symmetry and Ion-Pairing in Electron and Excitation Transfer, University of North Carolina, Chapel Hill, April 1995.

Electron and Excitation Transfer: MO Symmetry and Electrolyte Effects, Jackson State University, Jackson, March 1995.

Probing the Role of Molecular Orbital Symmetry and Electrolytes in Charge Transfer Reactions, University of Alabama, Tuscaloosa, February 1995. 
Symmetry and Electrolyte Effects in Intramolecular Electron Transfer, Department of Chemistry, University of Southern Mississippi, October 1994.

Transferring Electrons and Excitations, Department of Chemistry, Tulane University, March 1993.

Correlation Between Intramolecular Triplet Excitation, Electron and Hole Transfer Processes, Department of Chemistry, University of Bologna, Italy, January 1993.

Ionic Association in Intramolecular Electron Transfer, Department of Chemistry, University of Ferrara, Italy, January 1993.

Intramolecular Electron, Hole and Triplet Energy Transfer, Department of Chemistry, University of Perugia, Italy, January 1993.

Presentation at conferences (posters and talks):

Electrolyte Effects in Intramolecular Electron Transfer, 209th National ACS Meeting, Symposium on Complementarity of Photochemistry and Radiation Chemistry in the Study of Electron Transfer, Anaheim, California, April 1995

Low Lying Triplet States of Highly Twisted Olefins, 209th ACS National Meeting, Anaheim, California, April 1995

Ion Pairing with Photoinduced Charge Separated Species: Activation Energy Measurements, 209th ACS National Meeting, Anaheim, April 1995

Transient Charge Transfer Bands as Sensitive Probes of Ion-Pairing Processes, Electron Donor-Acceptor Interactions Gordon Research Conference, Newport, August 1994

Transient Charge Transfer Bands as Probes of Ionic Association Dynamics, 208th ACS National Meeting, Washington, August 1994

Ion-Pairing Effects in Intramolecular Electron Transfer Reactions: Distance and the Driving Force Dependence in the Weakly Exoergic Region, 10th International Conference on Photochemical Conversion and Storage of Solar Energy, Interlaken, Switzerland, July 1994

Vibronic Coupling and Electronic Energy Transfer in Bichromophoric Molecules, 10th International Conference on Photochemical Conversion and Storage of Solar Energy, Interlaken, Switzerland, July 1994

Electronic Coupling in Excited States and Cations of Donor-Acceptor Systems, 206th ACS National Meeting, Chicago, Illinois, August 1993 